\title{
CS Research Square \\ Relationship between the Height of Fibular Head and the Incidence and Severity of Knee Osteoarthritis
}

\section{Xinghui Xu}

Guangzhou University of Chinese Medicine

Jun Li

Guangzhou University of Chinese Medicine

\section{Deping Yao}

Guangzhou University of Chinese Medicine

\section{Pan Deng}

Guangzhou University of Chinese Medicine

\section{Boliang Chen}

Guangzhou University of Chinese Medicine

Yifei Liu

Guangzhou University of Chinese Medicine

\section{Yang jin ( $\nabla$ sxbjyangjin@163.com ) \\ Guangzhou University of Chinese Medicine}

\section{Research Article}

Keywords: Knee osteoarthritis,fibular head height,Kellgren-Lawrence grade,three-dimensional reconstruction,varus deformity

Posted Date: October 21st, 2021

DOI: https://doi.org/10.21203/rs.3.rs-986056/v1

License: () (1) This work is licensed under a Creative Commons Attribution 4.0 International License. Read Full License 


\section{Abstract}

Objective: To investigate the correlation between fibular head height and the incidence and severity of varus knee osteoarthritis based on three-dimensional reconstruction of the knee joint.

Methods: The data of knee joint imaging in our hospital from June 2018 to June 2020 were collected. The degree of varus deformity of the knee was assessed at the superior hip-knee-ankle angle of the X-rays. Three-dimensional reconstruction of patient computed tomography(CT)data was performed by mimics software. The fibular head height, joint line convergence angle (JLCA) and medial proximal tibial angle (MPTA) were measured in a threedimensional model. The patients were divided according to the Kellgren-Lawrence grade: group A: grade 0, group B: grade I, group C: grade II, group D: grade III, and group E: grade IV. The differences in age, gender, height, weight, body mass index(BMI), fibular head height, and degree of varus deformity (JLCA, MPTA, and coxa-knee-ankle angle) were compared. Ordinal multivariate logistic regression was used to analyze the correlation between fibular head height and Kellgren-Lawrence grade.Pearson correlation was used to analyze the correlation between fibular head height and Kellgren-Lawrence grade.

Results: 232 patients (232 knees) were finally included in the study, with Kellgren-Lawrence grades of 28 in group A, 31 in group B, 49 in group C, 53 in group D, and 71 in group E. The differences in age, gender, height, body mass index, fibular head height, JLCA, MPTA, and hip-knee-ankle angle among the five groups were statistically significant $(P<0.05)$, and the differences in body weight were not statistically significant $(P>0.05)$. There were significant differences in fibular head height, JLCA, JLCA and hip-knee-ankle angle between different groups $(P<0.05)$. Furthermore, there were significant differences in JLCA and hip-knee-ankle angle $(P<0.05)$, and both JLCA and hipknee-ankle angle increased with severe aggravation of Kellgren-Lawrence grade. Furthermore, both fibular head height and MPTA decreased as the Kellgren-Lawrence grade was severely aggravated. There was a significant negative correlation between Kellgren-Lawrence grade and fibular head height $(r=-0.812, P<0.001)$. Furthermore, there was a significant negative correlation between fibular head height and hip-knee-ankle angle $(r=-0.7905, P<$ 0.001). When Kellgren-Lawrence grade III and IV knees were considered as disease, ROC curve analysis showed a cut-off value of 10.63 for fibular head height and an AUC of 0.872 .

Conclusion: The height of fibular head in patients with varus knee osteoarthritis is smaller than that in nonosteoarthritis patients. In addition to body mass index, fibular head height is a risk factor for the pathogenesis of varus knee osteoarthritis, the smaller the fibular head height, the more severe the severity of osteoarthritis and the more severe the degree of varus deformity.

\section{Introduction}

Knee osteoarthritis, a degenerative knee disease-based disease, is a common cause of knee pain and lower limb disability. In the United States, half of the population may develop knee osteoarthritis at the age of 85 [1]. In addition, the study predicts that by 2030, the demand for total knee arthroplasty (TKA) will be 6 times that of 2005 [2].The medial compartment of the knee has an approximately 10 -fold higher risk of morbidity than the lateral compartment[3] and is generally thought to be associated with higher long-term pressures in the medial compartment of the knee [4]. For degenerative changes of the knee joint, degenerative changes in the medial compartment of patients with varus knee osteoarthritis are generally assessed clinically by X-rays, and changes in the lateral structure are usually overlooked. While Zhang Yingze's team found that there was an adaptive curve in the proximal fibula of patients with medial flip knee osteoarthritis [5],fibular weight bearing is one of the triggers of medial flip knee osteoarthritis, fibula bearing part of the body weight leads to greater force on the medial tibial 
plateau than on the lateral side, and long-term high load leads to faster collapse of the medial tibial plateau than on the lateral side, that is, the "uneven subsidence" theory [6].

We have also found in the clinic that not only is there an adaptive curvature of the proximal fibula in patients with introverted knee osteoarthritis, but the fibular head position is usually also high, even close to the level of the lateral tibial plateau. Our previous finite element studies found widening of the medial knee joint space and decreased medial compartment pressure after proximal fibular osteotomy (PFO)[7].Patients significantly relieved symptoms such as knee pain and limited mobility after PFO surgery[8]. Clinical practice and related studies have suggested that there may be an association between fibular head height and knee osteoarthritis. The change of fibular head height may be a manifestation of osteoarthritis or a risk factor for the occurrence and development of osteoarthritis. However, there is still a lack of relevant evidence-based evidence. X-ray are two-dimensional images and cannot assess the relative rotation between the tibia and fibula. In addition, the influence of knee rotation and flexion on the measurement results cannot be completely ruled out by using the knee X-ray film for measurement.

In this study, we measured fibular head height in a three-dimensional model of the knee joint. We hypothesized that fibular head height was associated with onset and severity of varus knee osteoarthritis. The purpose of this study was to investigate whether fibular head height is an independent risk factor for the pathogenesis of varus knee osteoarthritis,to investigate the correlation between fibular head height and imaging findings grading of varus knee osteoarthritis,and to investigate the correlation between fibular head height and the degree of varus deformity of the knee.

\section{Materials And Methods}

\section{General information}

Knee patients who visited Baoji Hospital of Traditional Chinese Medicine from June 2018 to June 2020 were retrospectively analyzed. Inclusion criteria $\triangle$ Knee arthritis patients or healthy volunteers. $\nabla$ Age $30-70$ years. $\otimes C T$ and $X$-ray image data were complete. Exclusion criteria $\nabla \otimes$ Previous history of lower limb trauma or surgery. $\nabla$ Suffering from congenital knee deformity or skeletal dysplasia. $\otimes$ Combined with diseases that seriously affect lower limb function, such as cerebral palsy, diabetic neuropathy, diabetic foot, etc. $\otimes$ Combined with other knee joint diseases, such as knee joint tumors, etc. All patients and their families gave informed consent to the treatment protocol, which was approved by the hospital ethics committee K.[2018] 038.

\section{Data Measurement}

Patient CT data were collected and three-dimensional reconstruction of the knee joint was performed by mimics software. Fibular head height: The distance between the upper edge of the fibular head and the horizontal tangent passing through the lowest point of the lateral tibial plateau was measured on a three-dimensional model of the knee (Figure 1A). Joint line convergence angle (JLCA): The angle between the articular surface of the distal femur and the plane of the tibial plateau based on a three-dimensional model of the knee joint (Figure 1B) [9]. Proximal medial tibial angle (MPTA): the medial angle between the tibial plateau plane and the mechanical axis of the tibia on the full-length anteroposterior X-ray of the standard weight-bearing lower limb (Figure 1C) [10]. Hip-knee-ankle angle: The angle between the extension line from the center of the femoral head to the center of the medial and lateral femoral condyles and the mechanical axis of the tibia on the full-length anteroposterior X-ray of the lower limb in the standard weight-bearing position, and the medial angulation is knee varus (Figure 1D). Kellgren-Lawrence grade: 
radiographic severity grading criteria for knee osteoarthritis, graded according to the findings of knee degeneration on X-ray: grade 0 , no significant abnormal changes on X-ray. grade I, suspected narrowing of the knee joint space or suspected osteophytes.grade II, significant osteophytes, mild narrowing of the joint space.grade III, moderate osteophytes, narrow joint space, mild subchondral osteosclerosis, small range.grade IV, a large number of osteophytes, severe narrowing of the joint space, significant osteosclerosis, accessible subchondral surface, accompanied by significant joint hypertrophy and deformity $[11,12]$.

Figure 1 Data measurement(A:Fibular head height is the distance between the fibular head edge and the horizontal tangent through the lowest point of the lateral tibial plateau B:JLCA: the angle between the tangent through the lower edge of the medial and lateral femoral condyles and the tibial plateau plane C:MPTA: the medial angle between the tangent through the tibial plateau and the tibial mechanical axis D:hip-knee-ankle angle: the angle between the extension line downward of the femoral mechanical axis (blue solid line) and the tibial mechanical axis (yellow solid line))

\section{Grouping}

The patients were divided into five groups according to the Kellgren-Lawrence grade of the knee: group A: grade 0, group B: grade I, group C: grade II, group D: grade III, and group E: grade IV.

\section{Statistical processing}

Statistical analysis was performed using SPSS 25.0 statistical software package (SPSS, USA). Age, height, weight, body mass index, fibular head height, JLCA, MPTA, and hip-knee-ankle angle were used as measurement data and conformed to the normal distribution by normal test, expressed as mean \pm standard deviation, and compared using analysis of variance and pairwise comparison q test,gender distribution was expressed as frequency, and compared using $\chi^{2}$ test. The test level $a$ is equal to 0.05 . Kellgren-Lawrence grade was used as the dependent variable, and age, gender, height, body mass index, and fibular head height were included as independent variables in ordinal multivariate logistic regression to analyze the effect of each factor on the occurrence and severity of knee osteoarthritis.Pearson correlation was used to analyze the correlation between fibular head height and hip-kneeankle angle and hip-knee-ankle angle, and correlation coefficient $r$ was used to reflect the degree of correlation between indicators.A receiver operating characteristic (ROC) curve was used for the evaluation of the cutoff point for the fibular head height.

\section{Results}

\section{Comparison of the general data of patients in different groups}

232 patients (232 knees) were finally included in the study, 86 males and 146 females, aged (61.12 \pm 10.98$)$ years (range 35-68 years). Kellgren-Lawrence grade was performed in 28 patients in group A, 31 patients in group B, 49 patients in group $C, 53$ patients in group $D$, and 71 patients in group $E$. The differences in age, gender, height, body mass index, fibular head height, JLCA, MPTA, and hip-knee-ankle angle among the five groups were statistically significant $(P<0.05)$, and the differences in body weight were not statistically significant $(P>0.05$, Table 1$)$. 
Comparison of the general data of patients in different groups

\begin{tabular}{|c|c|c|c|c|c|c|}
\hline Grouping & Cases & $\begin{array}{l}\text { Gender } \\
\text { \male/female } \rrbracket\end{array}$ & Age $\ y e a r s \rrbracket$ & Height $₫ \mathrm{~cm} \rrbracket$ & Weight $₫ \mathrm{~kg} \rrbracket$ & BMI $₫ \mathrm{~kg} / \mathrm{m}^{2} \square$ \\
\hline Group A & 28 & $11 / 17$ & $40.75 \pm 5.73$ & $160.07 \pm 4.88$ & $60.82 \pm 3.76$ & $23.79 \pm 1.86$ \\
\hline Group B & 31 & $13 / 18$ & $58.81 \pm 5.58 *$ & $160.45 \pm 7.29$ & $61.64 \pm 6.08$ & $24.09 \pm 3.38$ \\
\hline Group C & 49 & $18 / 31$ & $61.17 \pm 6.43^{\star} \#$ & $161.96 \pm 6.48$ & $62.15 \pm 5.46$ & $23.82 \pm 2.96$ \\
\hline Group D & 53 & $20 / 33$ & $62.17 \pm 6.72 * \#$ & $161.57 \pm 6.18$ & $62.41 \pm 6.47$ & $24.01 \pm 2.57$ \\
\hline Group E & 71 & $24 / 47$ & $65.46 \pm 7.12^{\star} \# \mathbf{\Delta} \nabla$ & $160.62 \pm 6.08$ & $65.47 \pm 6.48 * \# \mathbf{\Delta} \nabla$ & $25.45 \pm 3.04^{\star} \Delta \nabla$ \\
\hline statistics & - & $x^{2}=0.711$ & $F=304.969$ & $F=0.711$ & $F=5.291$ & $F=3.633$ \\
\hline P-values & - & 0.950 & 0.000 & 0.585 & 0.000 & 0.007 \\
\hline
\end{tabular}

\section{Comparison of imaging measurement indicators between different groups}

There were significant differences in fibular head height, JLCA, MPTA and hip-knee-ankle angle between different groups $(P<0.05)$. Furthermore, there were significant differences in JLCA and hip-knee-ankle angle $(P<0.05)$, and both JLCA and hip-knee-ankle angle increased with severe aggravation of Kellgren-Lawrence grade. Furthermore, both fibular head height and MPTA decreased as the Kellgren-Lawrence grade was severely aggravated. There was no significant difference in fibular head height and MPTA between group $B$ and group $C(P>0.05)$, but there were significant differences in fibular head height and other MPTA $(P<0.05$ Table 2$)$.

Table 2

Comparison of imaging measurement indicators between different groups 


\begin{tabular}{|c|c|c|c|c|c|}
\hline Grouping & Cases & $\begin{array}{l}\text { Fibular head height } \\
\rrbracket \mathrm{mm} \rrbracket\end{array}$ & $\mathrm{JLCA}^{\circ} \rrbracket$ & MPTA $\rrbracket^{\circ} \rrbracket$ & $\begin{array}{l}\text { Hip-knee-ankle Angle } \\
\square^{\circ} \square\end{array}$ \\
\hline Group A & 28 & $13.34 \pm 1.61$ & $0.54 \pm 0.09$ & $87.93 \pm 1.05$ & $1.86 \pm 0.16$ \\
\hline Group B & 31 & $11.78 \pm 1.34^{\star}$ & $0.88 \pm 0.16^{\star}$ & $86.37 \pm 1.67 *$ & $3.16 \pm 0.324^{\star}$ \\
\hline Group C & 49 & $11.46 \pm 1.27 *$ & $1.73 \pm 0.12^{\star} \#$ & $86.21 \pm 1.49 *$ & $4.43 \pm 0.67 * \#$ \\
\hline Group D & 53 & $10.07 \pm 1.20 * \# \boldsymbol{\Lambda}$ & $3.46 \pm 0.34 * \# \boldsymbol{\Delta}$ & $86.06 \pm 1.70 *$ & $5.49 \pm 1.11 * \# \boldsymbol{\Delta}$ \\
\hline Group E & 71 & $7.46 \pm 1.24 * \# \mathbf{\Delta} \nabla$ & $6.49 \pm 0.86 * \# \mathbf{\Delta} \nabla$ & $84.46 \pm 1.07 * \# \mathbf{\Delta} \nabla$ & $10.33 \pm 1.08 * \# \mathbf{\Delta} \nabla$ \\
\hline statistics & - & $F=142.173$ & $F=179.437$ & $F=34.432$ & $F=725.932$ \\
\hline P-values & - & $<0.001$ & $<0.001$ & $<0.001$ & $<0.001$ \\
\hline
\end{tabular}

\section{Correlation between fibular head height and knee joint severity and varus deformity}

There was a significant negative correlation between Kellgren-Lawrence grade and fibular head height $(r=-0.812, \mathrm{P}<$ $0.001)$, that is, the more severe the Kellgren-Lawrence grade, the lower the fibular head height. Furthermore, there was a significant negative relationship between fibular head height and coxa-knee-ankle angle $(r=-0.7905, P<0.001)$, that is, the more severe the grade of knee varus deformity, the lower the fibular head height (Figure 2).

\section{Analysis of influencing factors of Kellgren-Lawrence grading}

The effects of age, gender, height, weight, body mass index, and fibular head height on Kellgren-Lawrence grade of varus knee osteoarthritis were analyzed using ordinal logistic regression that met the proportional odds hypothesis. The results of the parallel line test suggested that the proportional odds hypothesis of the regression model was established and it was suitable to use ordinal logistic regression analysis $\left(X^{2}=379.95, P=0.000\right)$, the pseudo $\mathrm{R}^{2}$ of the model $=0.806$, suggesting that the statistical model fitted well. The results showed that fibular head height and body mass index had a significant effect on Kellgren-Lawrence grade, and their ORs $(95 \% \mathrm{Cl})$ were $0.745(-1.294,-0.862)$ and $1.246(-2.781,-0.568)$, respectively. It is suggested that for every $1 \mathrm{~mm}$ increase in fibular head height, the risk of developing osteoarthritis or a 1 grade increase in Kellgren-Lawrence grade is reduced to 0.745 . For each $1 \mathrm{~kg} / \mathrm{m}^{2}$ increase in body mass index, the risk of developing osteoarthritis or a 1 -grade increase in the Kellgren-Lawrence grade increased by 1.246 -fold (Table 3 ).

Table 3

Ordinal logistic regression with Kellgren-Lawrence grade as the dependent variable 


\begin{tabular}{|lllllll|}
\hline Parameters & $\beta$ & $S . E$ & Wals & $P$ & OR & $95 \% \mathrm{Cl}$ \\
\hline Fibular head height & -1.078 & 0.110 & 95.645 & 0.000 & 0.745 & $-1.294,-0.862$ \\
\hline Age & 0.318 & 0.038 & 68.741 & 0.053 & 1.134 & $0.243,0.394$ \\
\hline Height & -.524 & 0.174 & 9.042 & 0.432 & 1.045 & $-0.865,-0.182$ \\
\hline Weight & 0.672 & 0.221 & 9.230 & 0.534 & 0.895 & $0.238,1.105$ \\
\hline BMI & 0.325 & 0.565 & 8.795 & 0.019 & 1.246 & $-2.781,-0.568$ \\
\hline Gender & -0.382 & 0.316 & 1.462 & 0.227 & 1.023 & $-1.002,0.237$ \\
\hline Note: $\beta$ : regression coefficient,SE: standard error of regression coefficient,OR: odds ratio,Cl: confidence interval \\
\hline
\end{tabular}

\section{ROC curve analysis}

When Kellgren-Lawrence grade III and IV knees were considered as disease and normal controls for knees below grade III, ROC curve analysis showed a cutoff value of 10.63 for fibular head height and an AUC of 0.872. In addition, when Kellgren-Lawrence grade IV knees were considered as disease and normal controls of knees below grade IV, ROC curve analysis showed a cutoff value of 8.25 for fibular head height and an AUC of 0.599 (Table 4 and Figure 3).

Table 4

ROC curve analysis

\begin{tabular}{|c|c|c|c|c|c|c|c|c|}
\hline \multirow[t]{2}{*}{ Parameters } & \multirow[t]{2}{*}{ disease } & \multirow[t]{2}{*}{ Area } & \multirow[t]{2}{*}{$P$} & \multicolumn{2}{|c|}{ Progressive $95 \% \mathrm{Cl}$} & \multirow{2}{*}{$\begin{array}{l}\text { Cutoff } \\
\text { value }\end{array}$} & \multirow[t]{2}{*}{ Sensibility } & \multirow[t]{2}{*}{ Specificity } \\
\hline & & & & $\begin{array}{l}\text { Lower } \\
\text { limit }\end{array}$ & $\begin{array}{l}\text { Upper } \\
\text { limit }\end{array}$ & & & \\
\hline
\end{tabular}

Fibular head height

\mm》

$\begin{array}{lll}\quad & \quad 0.872 & 0.000\end{array}$

IV $\mathbb{Z}$

Fibular head height $\quad$ IIV $\bigotimes \mathrm{mm} \rrbracket$
$0.826 \quad 0.918$

$10.63 \quad 83.9 \% \quad 80.6 \%$

$0.848 \quad 0.915$
$0.899 \quad 0.000$
8.25
$81.7 \%$
$95.7 \%$

\section{Discussion}

This study innovatively measured image data by three-dimensional reconstruction of the knee and found a correlation between fibular head height and osteoarthritis of the flipped knee. First, the fibular head height of patients with varus knee arthritis was smaller than that of non-osteoarthritis population, and the fibular head height was a risk factor for osteoarthritis in addition to body mass index. Second, the fibular head height was negatively correlated with the severity of osteoarthritis and the degree of varus deformity.

The fibular head is an important ligament and tendon attachment point, including the lateral collateral ligament, arcuate ligament, popliteofibular ligament, biceps femoris muscle, and peroneus longus muscle, and the popliteal tendon is indirectly connected to it through the popliteofibular ligament, so its location is closely related to the 
function of the ligament and tendon[13]. Biomechanical studies have found that compared with the normal population, patients with varus knee osteoarthritis have a higher peak activity level of the peroneus longus muscle and a lower peak activity level of the biceps femoris muscle,while on the first day after PFO, the activity level of the peroneus longus muscle is significantly lower while the peroneus head is shifted downward, and the activity level of the biceps femoris muscle is significantly increased at 6 months after surgery and maintained at a higher level [5]. Theoretically, a decrease in the height of the fibular head will lead to relaxation and tension reduction of the lateral knee related ligaments and tendons, and muscles and ligaments are important structures to maintain knee stability[14]. This study found that for every $1 \mathrm{~mm}$ increase in fibular head height, the risk of developing osteoarthritis or a 1 grade increase in the Kellgren-Lawrence grade was reduced to 0.745 . Showed that changes in fibular head height in internal turned knee osteoarthritis are not only manifestations of osteoarthritis, but also more likely to be a risk factor leading to knee instability, arthritis pathogenesis and progression.

Lateral soft tissues of the knee play an important role in limiting abnormal external rotation of the tibia [15]. The posterolateral knee ligament complex, composed of the lateral collateral ligament, arcuate ligament, popliteofibular ligament, and popliteal tendon, produces the greatest limiting force against external tibial rotation when the knee is flexed $30^{\circ}-45^{\circ}[16]$. The maximum limiting force against external tibial rotation at $0^{\circ}-30^{\circ}$ of knee flexion is generated by the lateral collateral ligament,the popliteal complex plays a major role in place when the knee continues to flex [17]. This study confirmed that fibular head height was negatively correlated with the severity of varus knee osteoarthritis, that is, the lower the fibular head height, the more severe the severity of knee osteoarthritis. Elevated relative position of the fibular head in patients with inversion osteoarthritis will cause a decrease in lateral soft tissue tension, so instability of the knee in the horizontal position may manifest as abnormal external rotation of the tibia. It has been confirmed that there is indeed an abnormal increase in the external rotation angle of the tibia in patients with osteoarthritis [18-19], and the abnormal external rotation angle of the tibia increases with the severity of arthritis [20]. These literatures also confirmed the findings of the present study. Abnormal external rotation of the tibia is an important cause of increased pressure in the medial knee compartment [21, 22], while abnormal increase in pressure will exacerbate knee degeneration, such as hyperosteogeny and osteosclerosis [23].

The lateral soft tissues of the knee joint play an important role in maintaining the normal lower limb alignment [2426], and the posterolateral ligamentous complex of the knee joint is the main structure that limits varus deformity of the knee joint. The lateral collateral ligament plays a major role in limiting varus deformity of the knee when the knee is flexed from $0^{\circ}$ to $30^{\circ}$, and the biceps femoris plays a major role when flexion exceeds $30^{\circ}$ [27]. As the attachment point of the above ligaments and tendons, the relative upward movement of the position of the fibular head may weaken the structural function that limits knee varus, and the worse the stability of the lateral structure of the knee in the coronal plane, the more severe the varus deformity performance. This may be part of the mechanism by which the degree of varus deformity in varus knee osteoarthritis is inversely correlated with fibular head height. Some scholars believe that the force on the fibular head in the vertical direction is a tensile force rather than a compressive force, which may be the mechanical reason for the displacement of the fibular head. However, this study confirmed that in addition to the mechanism by which the fibula affects the pathogenesis and severity of varus knee osteoarthritis, there is a close association with fibular head height.

This study innovatively measured image data by three-dimensional reconstruction of the knee and found a correlation between fibular head height and osteoarthritis of the flipped knee. This study then remains limited. First of all, this study was a single-center study with a limited number of cases and possible selection bias. Second, this study was retrospective and could not describe the dynamic changes in fibular head height and progression of 
osteoarthritis in each patient. In addition, the effect of fibular head height on knee osteoarthritis still requires mechanical experiments such as finite element for mechanistic studies.

In conclusion, the height of fibular head in patients with varus knee osteoarthritis is smaller than that in nonosteoarthritis patients. In addition to body mass index, fibular head height is a risk factor for the pathogenesis of varus knee osteoarthritis, the smaller the fibular head height, the more severe the severity of osteoarthritis and the more severe the degree of varus deformity.

\section{Abbreviations}

PFO:proximal fibular osteotomy;CT:computed tomography;joint line convergence angle (JLCA); medial proximal tibial angle (MPTA);BMI:body mass index; total knee arthroplasty (TKA)

\section{Declarations}

\section{Acknowledgement}

Grateful acknowledgement is made to my supervisor Professor He Wei who gave me considerable help by means of suggestion, comments and criticism. In addition, I deeply appreciate the contribution to this thesis made in various ways by my friends and classmates.

\section{Funding}

Publication charges for this article were funded by Natural Science Foundation of China (81873327), Natural Science Foundation of Guangdong (2015A030313353), Science and Technology Project of Guangzhou (201510010228).

\section{Availability of data and materials}

All the data will be available upon motivated request to the corresponding author of the present paper.

\section{Author Contributions}

Xinghui Xu was responsible for the design and implementation of the study presented. Jin Yang and Xinghui Xu were responsible for the knee joint 3D reconstruction.Jun Li,Yifei Liu, Pan Deng,Boliang Chen and Deping Yao conducted the experiments and were responsible for the acquisition of the data. Xinghui Xu prepared the initial draft of the manuscript. Jin Yang gave critical feedback during the study and critically revised the submitted manuscript for important ntellectual content. All authors have read and approved the final manuscript to be submitted.

\section{Ethical approval}


This study was conducted in agreement with the Declaration of Helsinki and its later amendments or comparable ethical standards and had been approved by the ethics board of Baoji Hospital of Traditional Chinese Medicine(No: K.[2018]038).

\section{Consent for publication}

Not applicable

\section{Competing interests}

The authors declare no competing interests.

\section{References}

1. Murphy Louise,Schwartz Todd A,Helmick Charles G et al. Lifetime risk of symptomatic knee osteoarthritis. [J].Arthritis Rheum, 2008, 59: 1207-13.

2. Kurtz Steven, Ong Kevin,Lau Edmund et al. Projections of primary and revision hip and knee arthroplasty in the United States from 2005 to 2030.[J].J Bone Joint Surg Am, 2007, 89: 780-5.

3. Andersen Jane Dahl,Folkestad Lars,Hald Jannie Dahl et al. Osteoarthritis in Osteogenesis Imperfecta: A Nationwide Register-based Cohort Study.[J].Bone, 2021, undefined: 116222.

4. NieY, MaJ, HuangZ, et al. Upper partial fibulectomy improves knee biomechanics and function and decreases knee pain of osteoarthritis: a pilot and biomechanical study[J]. J Biomech, 2018, 71: 22-29.

5. WangJ, $L v H Z$, ChenW, et al. Anatomical adaptation of fibula and its mechanism of proximal partial fibulectomy associated with medial compartment knee osteoarthritis[J]. Orthop Surg, 2019, 11(2): 204-211.

6. DongT, ChenW, ZhangF, et al. Radiographic measures of settlement phenomenon in patients with medial compartment knee osteoarthritis[J]. Clin Rheumatol, 2016, 35(6): 1573-1578.

7. Pan Deng, TianYe Lin,Peng Yang et al. Effects of proximal fibular osteotomy on stress changes in mild knee osteoarthritis with varus deformity. a finite element analysis.[J].J Orthop Surg Res, 2020, 15: 375.

8. QinD, ChenW, WangJ, et al. Mechanism and influencing factors of proximal fibular osteotomy for treatment of medial compartment knee osteoarthritis: a prospective study[J]. J Int Med Res, 2018, 46(8): 3114-3123.

9. LeeDH, ParkSC, ParkHJ, et al. Effect of soft tissue laxity of the knee joint on limb alignment correction in openwedge high tibial osteotomy[J]. Knee Surg Sports Traumatol Arthrosc, 2016, 24(12): 3704-3712.

10. PornrattanamaneewongC, NarkbunnamR, ChareancholvanichK. Medial proximal tibial angle after medial opening wedge HTO: a retrospective diagnostic test study[J]. Indian J Orthop, 2012, 46(5): 525-530.

11. Baker Breanne S,Bozynski Chantelle C,Leary Emily V et al. Tibial Bone Quality in Former Bariatric Surgery Patients with Osteoarthritis.[J]. Obes Surg, 2021, 36(2): 123-133.

12. PeterssonIF, BoegårdT, SaxneT, et al. Radiographic osteoarthritis of the knee classified by the Ahlbäck and Kellgren \& Lawrence systems for the tibiofemoral joint in people aged 35-54 years with chronic knee pain[J]. Ann Rheum Dis, 1997, 56(8): 493-496. 
13. van Kuijk K S R,Reijman M,Bierma-Zeinstra S M A et al. Posterior cruciate ligament injury is influenced by intercondylar shape and size of tibial eminence.[J].Bone Joint J, 2019, null: 1058-1062.

14. Tian Jidong,Wang Zhiyong,An Jie et al. Arthroscopic debridement combined with proximal fibular osteotomy in medial tibial articular genu osteoarthritis treatment. systematic review and meta-analysis.[J].Ann Palliat Med, 2021, 10: 7894-7904.

15. Drenck Tobias C,Preiss Achim,Domnick Christoph et al. The Popliteus Bypass provides superior biomechanical properties compared to the Larson technique in the reconstruction of combined posterolateral corner and posterior cruciate ligament injury.[J].Knee Surg Sports Traumatol Arthrosc, 2021, 29: 732-741.

16. VapAR, SchonJM, MoatsheG, et al. The role of the peripheral passive rotation stabilizers of the knee with intact collateral and cruciate ligaments: a biomechanical study[J]. Orthop J Sports Med, 2017, 5(5): 2325967117708190.

17. Figueroa Francisco,Figueroa David,Putnis Sven et al. Posterolateral corner knee injuries: a narrative review. [J].EFORT Open Rev, 2021, 6: 676-685.

18. PagnanoMW, HanssenAD. Varus tibial joint line obliquity. a potential cause of femoral component malrotation[J]. Clin Orthop Relat Res, 2001(392): 68-74.

19. Ries Christian,Boese Christoph Kolja,Ott Nadine et al. Intra-individual variance of bilateral femoro-tibial leg rotation: a CT study of 105 healthy subjects.[J].Knee Surg Sports Traumatol Arthrosc, 2021, 29: 1106-1113.

20. MatsuiY, KadoyaY, UeharaK, et al. Rotational deformity in varus osteoarthritis of the knee: analysis with computed tomography[J]. Clin Orthop Relat Res, 2005(433): 147-151.

21. KenaweyM, LiodakisE, KrettekC, et al. Effect of the lower limb rotational alignment on tibiofemoral contact pressure[J]. Knee Surg Sports Traumatol Arthrosc, 2011, 19(11): 1851-1859.

22. YazdiH, MallakzadehM, Sadat FarshidfarS, et al. The effect of tibial rotation on knee medial and lateral compartment contact pressure[J].Knee Surg Sports Traumatol Arthrosc, 2016, 24(1): 79-83.

23. ThorpLE, WimmerMA, BlockJA, et al. Bone mineral density in the proximal tibia varies as a function of static alignment and knee adduction angular momentum in individuals with medial knee osteoarthritis[J]. Bone, 2006, 39(5): 1116-1122.

24. VeltriDM, DengXH, TorzilliPA, et al. The role of the cruciate and posterolateral ligaments in stability of the knee. $A$ biomechanical study[J]. Am J Sports Med, 1995, 23(4): 436-443.

25. Liu Ming-Yang,Wang Hai-Bo,Liu Shi-Wei et al. Three-point Method to Guide the Tibial Resection and Component Placing in Total Knee Arthroplasty.[J].Orthop Surg, 2020, 12: 861-868.

26. LaPradeRF, ResigS, WentorfF, et al. The effects of grade III posterolateral knee complex injuries on anterior cruciate ligament graft force. A biomechanical analysis[J]. Am J Sports Med, 1999, 27(4): 469-475.

27. Malalagama Geethal N,Solomon Lucian B,Loftus William K,Complete tibial insertion of the biceps femoris tendon: an MRI-based study to assess the prevalence of this variant.[J].Surg Radiol Anat, 2020, 42: 661-665.

\section{Figures}




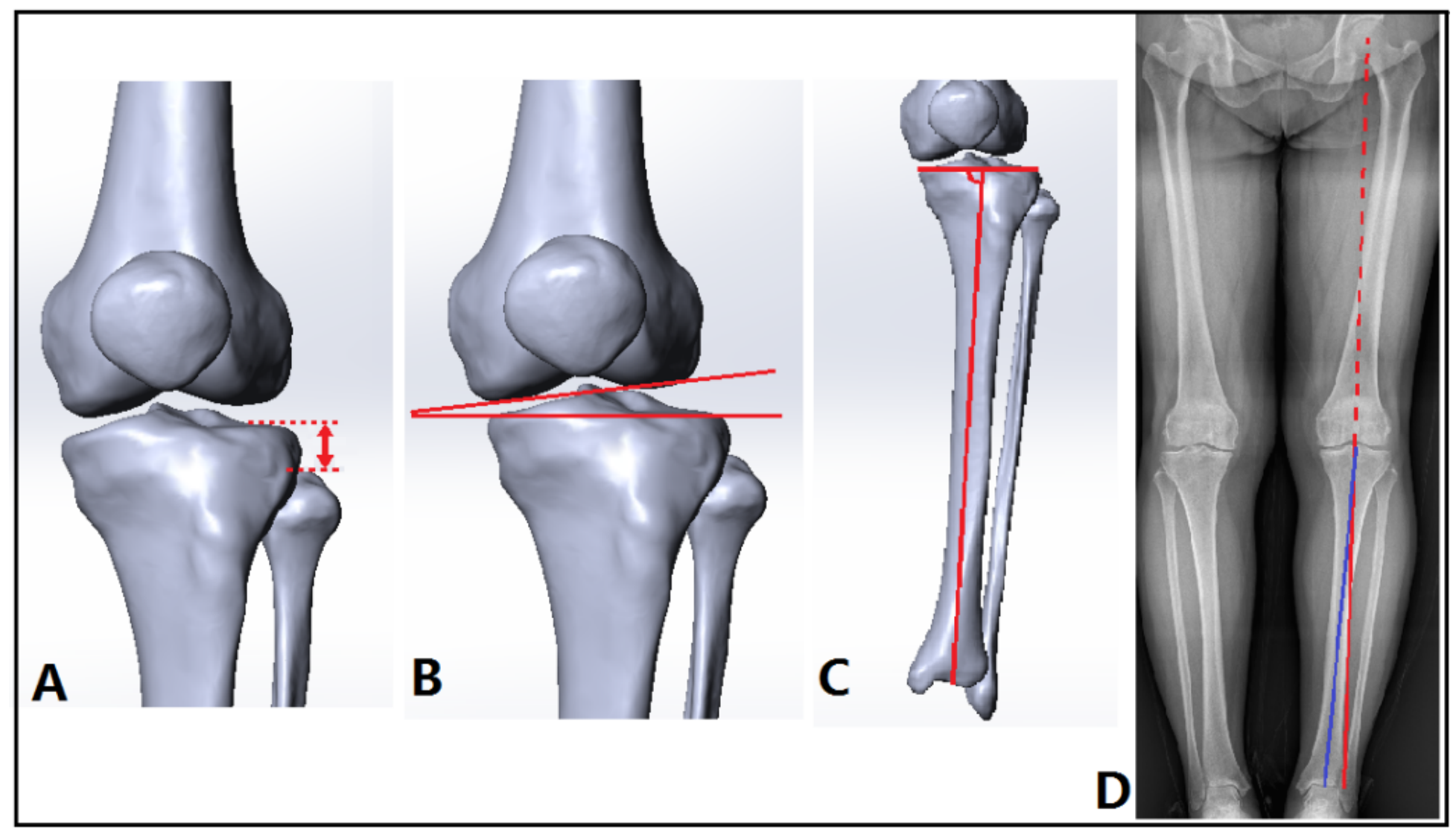

Figure 1

Data measurement(A:Fibular head height is the distance between the fibular head edge and the horizontal tangent through the lowest point of the lateral tibial plateau B:JLCA: the angle between the tangent through the lower edge of the medial and lateral femoral condyles and the tibial plateau plane C:MPTA: the medial angle between the tangent through the tibial plateau and the tibial mechanical axis D:hip-knee-ankle angle: the angle between the extension line downward of the femoral mechanical axis (blue solid line) and the tibial mechanical axis (yellow solid line))

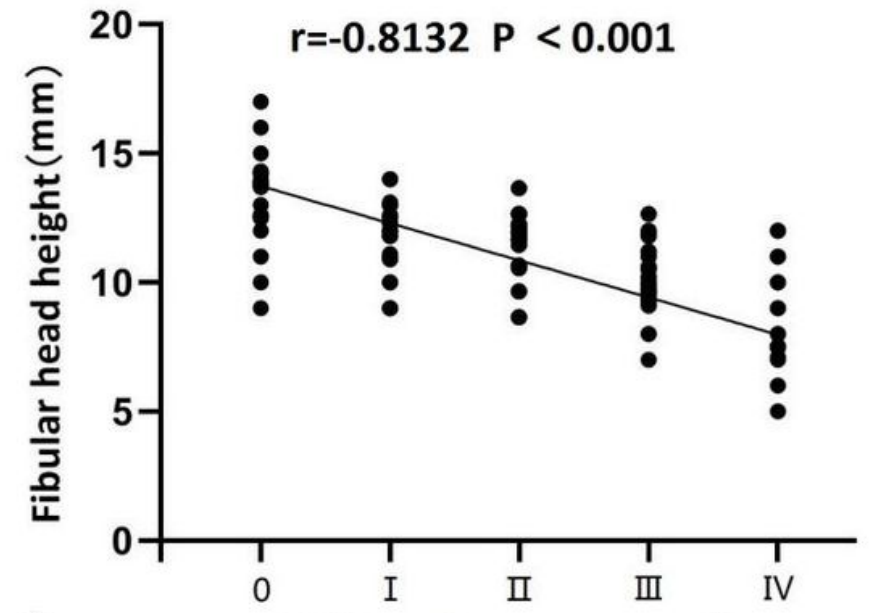

A

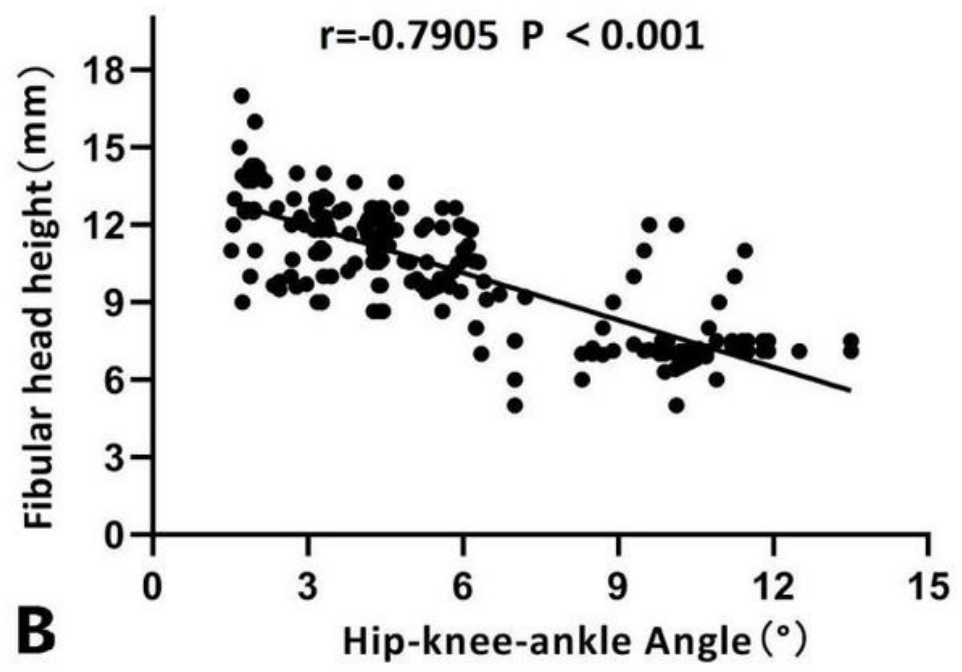

Figure 2

Correlation between fibular head height and knee joint severity and varus deformity 
Kellgren-Lawrence III and IV
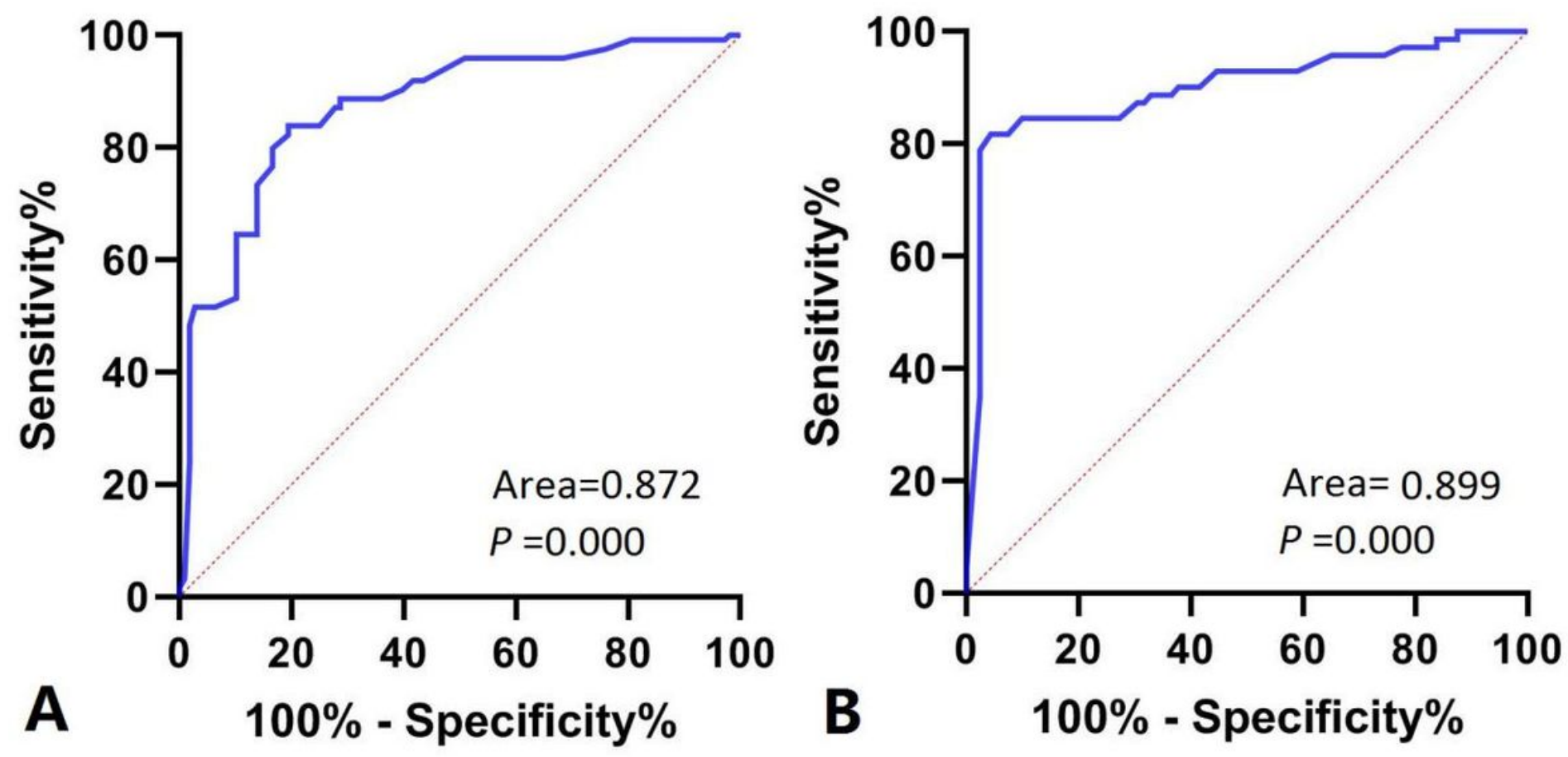

Figure 3

ROC curve analysis (A:Kellgren-Lawrence grade III and IV,B:Kellgren-Lawrence grade IV) 